\title{
MONITORING POPULATION EVOLUTION IN THE PEARL RIVER DELTA FROM 2000 TO 2010
}

\author{
Sisi Yu ${ }^{1,2}$, Fang Liu ${ }^{1, *}$, Zengxiang Zhang ${ }^{1}$ \\ 1 Institute of Remote Sensing and Digital Earth, Chinese Academy of Sciences, Beijing 100101, China - (yuss2017, liufang, \\ zhangzx)@radi. ac. cn \\ ${ }^{2}$ University of Chinese Academy of Sciences, Beijing 100049, China
}

KEY WORDS: DMSP/OLS, Population Spatialization, Time Series, Remote Sensing, The Pearl River Delta, Population Evolution

\begin{abstract}
:
On behalf of more populous and developed regions in China, urban agglomerations have become important carries loading active economic activities and generous social benefits, and experienced sharper population increase, which results in great threat on local eco-environment construction. Therefore, exact and detailed population monitoring and analyzing, especially on the long sequence and multi frequency, is of great significance. The nighttime light time-series (NLT) products has been proven to be one of the most useful remotely sensed imagery to acquire persons at $1 \mathrm{~km} * 1 \mathrm{~km}$ scales. However, the existed problems, such as light saturation and blooming, greatly limit the accuracy of estimated results. Furthermore, it's difficult to spatialize population at $\mathrm{km}^{2}$ level due to the lack of basic data in non-census years. In order to solve all problems mentioned above, the populous Pearl River Delta was selected as the study area. A new residential extent extraction index (REEI) was proposed to solve light saturation and blooming problems. Population spatialization methods in census and non-census years were applied to acquire detailed population distribution from 2000 to 2010. Results showed the feasibility of the proposed methods in this work. During the decade, population was denser in the central PRD and sparser in the eastern, western and northern PRD. The speed of population increase was various in nine cities, but faster in 2000-2005 than 2005-2010.
\end{abstract}

\section{INTRODUCTION}

Population distribution and dynamics, especially in populous area like urban agglomerations, lead to great influence on local socio-economic development and ecological environmental changes. Detailed information of population distribution and dynamics is of great significance on exploring the relationship between human activities and urbanization, land use changes, as well as the ecological effects (Yu et al., 2018). However, the most authoritative and reliable population datasets in China we can get are census data, which cannot describe the internal differences of population distribution in each administrative unit (Zeng et al., 2011). Hence, a feasible method to spatialize population to more detailed units is necessary.

Owing to the special capacity in reflecting human activities at night, the nighttime lights time-series (NLT) image products of Defense Meteorological Satellite Program's Operational Linescan System (DMSP/OLS) have been widely applied to population density simulation (Sun et al., 2017; Tripathy et al., 2017). Population spatialization method based on NLT products provides effective approach to monitor population distribution and analyze population evolution, which demonstrates spatial heterogeneity of population distribution at $1 \mathrm{~km} \times 1 \mathrm{~km}$ scales. However, the existing materials cannot provide detailed population distribution information especially in non-census years like 2005. Additionally, there are still several limitations for monitoring population evolution using NLT products, such as geometric errors, incompatibility of Digital Number (DN) values, light saturation and blooming, and so on (Elvidge et al., 1999; Zhang et al., 2013; Zhao et al., 2014).
At present, there are numbers of studies accomplished population spatialization using corrected NLT products. Most scholars, such as Sutton (1997), Lo (2001), Zhuo et al. (2009), Zeng et al. (2011) and Yu et al. (2018), are devoted to studying population spatialization methods at national or regional scales using NLT products. However, studies on population distribution in populous areas like urban agglomerations are relatively scarce. Moreover, more scholars show strong interesting in population evolution and have tried to explain the evolution rules of population using various statistics at national, provincial or county's scales (Yang et al., 2016). Nevertheless, they rarely explore population evolution differences between various cities using detailed population distribution data, especially at pixel scales.

On behalf of one major urban agglomeration in China, the PRD has experienced highintensity development, and population increase in this region is quirt rapid, resulting in increasing tension on local socio-economic development and environmental protection (Wei et al., 2017). This study aims to describe the detailed population evolution in the PRD, reveal its characteristics from the spatial and temporal aspects.

\section{MAIN BODY}

\subsection{Study Area}

In this work, the study area mainly contains nine cities in the PRD, including Guangzhou, Shenzhen, Zhuhai, Zhaoqing, Huizhou, Zhongshan, Foshan, Dongguan and Jiangmen (Figure $1)$.

\footnotetext{
* Corresponding author
} 
The PRD is located in the south-central Guangzhou Province, across the estuary of the Pearl River and next to the Southeast Asia, from $21^{\circ} \mathrm{N}$ to $24^{\circ} \mathrm{N}$ and from $112^{\circ} \mathrm{E}$ to $115^{\circ} \mathrm{E}$. The special geographic location brings humid subtropical climate and convenient traffic condition, which is suitable for human life and activities (Wei et al., 2017). In addition, the PRD experienced rapid economic development, society advancement and commerce prosperity owing to the proposed reform and opening policies in 1978 (You et al., 2013). Nowadays, the PRD has become one of the most populous regions in China. Meanwhile, the PRD is also one of the largest mega-region in the world in terms of both surface area and population (the World Bank Group, 2015).

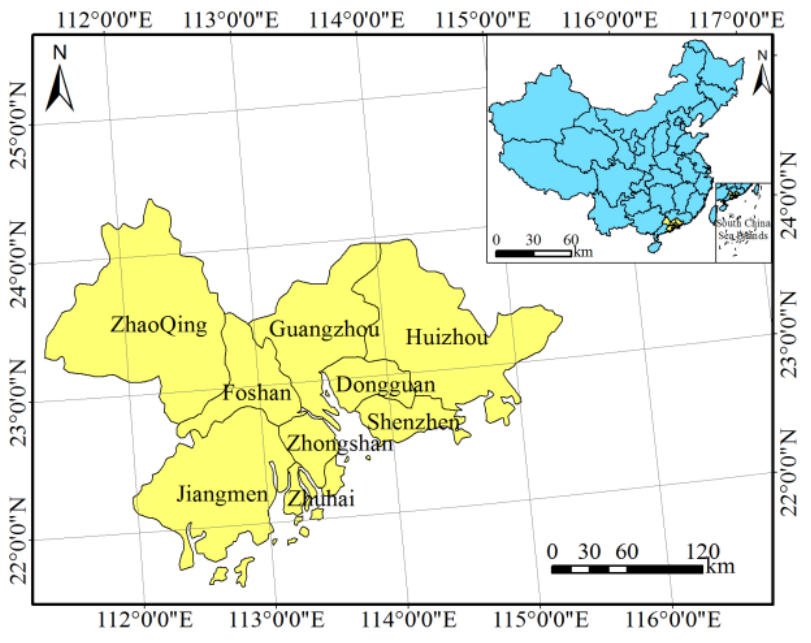

Figure 1. Study area distribution

\subsection{Materials and Pre-processing}

\begin{tabular}{|l|l|l|l|}
\hline Products & Time & Scenes & Resolution \\
\hline NLT & 2000 & 1 & \\
& 2005 & 1 & $\begin{array}{l}\text { 30 arc second } \\
\text { grids }\end{array}$ \\
& 2010 & 1 & \\
MOD09A1 & $2000-9$ & 4 & \\
& $2005-9$ & 4 & $500 \mathrm{~m}$ \\
Population & $2010-9$ & 4 & \\
Boundary & 2000 & & \\
Vector & & - & \\
\hline
\end{tabular}

Table 1. Datasets used in this study

Totally 15 remotely sensed images were applied to monitor population evolution in the PRD, of which $20 \%$ and $80 \%$ were NLT products from the DMSP/OLS and MOD09A1 8d composite products from NASA, respectively (Table 1). The spatial resolutions range from nearly $500 \mathrm{~m}$ to 30 arc second grids, and fully met the needs of this work by using visible bands, near-infrared band and shortwave infrared band.

Additionally, the administrative boundary of nine cities in the PRD was obtained from the Data Center for Resources and Environmental Sciences, Chinese Academy of Sciences. The referenced population in 2000, 2005 and 2010 were acquired from the fifth census bulletin, $1 \%$ population sample surveys and the sixth census bulletin, respectively.

During the data pre-processing, all remotely sensed images were projected to Asia North Albers Equal Area Conic (AN_Albers) coordinate system and resampled to $1 \mathrm{~km}$ spatial resolution. The NLT products were inter-calibrated using the method proposed by Elvidge et al. (1999) and geometric corrected according to Zhao et al. (2014).

\subsection{Methodology}

The experimental process can be summarized into three steps. Firstly, a new model named the residential extent extraction index (REEI) was employed to impair light saturation and blooming in this work. Secondly, the optimal regression functions in 2000 and 2010 were built based on total DN values and census data of each county in the PRD. Then optimal regression functions were applied to NLT products in the PRD and the initial population at pixel scales in 2000 and 2010 were simulated. In addition, the initial population distribution maps were modified by appropriate proportionality coefficients. Thirdly, in accordance with population distribution maps in 2000 and 2010 and population growth model proposed by Malthus (2008), population distribution in 2005 was calculated.

2.3.1 The Residential Extent Extraction Index: On basis of the distribution characteristics of vegetation, water, and residential buildings, the residential extent extraction index (REEI) was proposed to solve light saturation and blooming problems (Function (1)).

$$
\text { REEI }=\frac{(1-\mid \text { NDVI } \mid) \cdot(1+\mathrm{MNDBI})}{1+\mathrm{MNDWI}} \cdot \mathrm{NLT}_{\mathrm{DN}}
$$

where NDVI is the average normalized difference vegetation index (NDVI) value calculated from the MOD09A1 products; MNDBI is the average modified normalized difference barren index (MNDBI) value calculated from the MOD09A1 products the MNDWI are the average modified normalized difference water index (MNDWI) value calculated from the MOD09A1 products; $\mathrm{NLT}_{\mathrm{DN}}$ indicates the DN values of the NLT products.

2.3.2 Population Spatialization in Census Years: Population in 2000 and 2010 were acquired from the fifth and sixth census bulletin, by which the statistical data of population in each city even county should be exact and specific. Hence, it is relatively easy to spatialize persons in 2000 and 2010 to $1 \mathrm{~km} * 1 \mathrm{~km}$ based on the population spatialization method proposed before (Yu et al., 2018). Firstly, the polynomial regression functions were built according to the relationship between the total population and sum DN values at county level. Then, the polynomial regression functions would be applied to NLT products.

$$
\begin{gathered}
\mathrm{T}_{-} \mathrm{Pop}_{\mathrm{i}}=\mathrm{f}\left(\mathrm{TDN}_{\mathrm{j}}\right) \\
\mathrm{f}=\mathrm{a} * \mathrm{NLT}_{\mathrm{DN}}{ }^{3}+\mathrm{b} * \mathrm{NLT}_{\mathrm{DN}}{ }^{2}+\mathrm{c} * \mathrm{NLT}_{\mathrm{DN}}
\end{gathered}
$$

where T_Pop $_{i}$ is the sum population in the $\mathrm{jth}$ county; TDN $_{j}$ is the total $\mathrm{DN}$ values in the $\mathrm{jth}$ county; $\mathrm{f}$ indicates the regression functions between $\mathrm{T}_{-} \mathrm{Pop}_{\mathrm{i}}$ and $\mathrm{TDN}_{\mathrm{j}} ; \mathrm{a}, \mathrm{b}$ and $\mathrm{c}$ are coefficients; NLT $_{\mathrm{DN}}$ indicates the DN values of the NLT products. Finally, as the initial results of Function (2) and Function (3), an adjustment using proportionality coefficient should be executed. 
2.3.3 Population Spatialization in Non-census Years: Compared with population spatialization in census years like 2000 and 2010, it's more difficult to spatialize persons in non-census years (i.e., 2005) to limited scales as $1 \mathrm{~km} * 1 \mathrm{~km}$ due to the deficiency of detailed census data. Therefore, in this study, the population theory (Malthus, 2008) was applied to the NLT product in 2005 (Function (4)).

$$
\begin{gathered}
P_{2005}=\mathrm{K}_{2005} * \mathrm{NLT}_{D N} \\
\mathrm{~K}_{2005}=\mathrm{K}_{2000} \cdot(1+\mathrm{r})^{5}
\end{gathered}
$$

where Pop Po05 is the estimated population in 2005; $\mathrm{K}_{2005}$ is the ratio between population and $\mathrm{DN}$ values in 2005 ; $\mathrm{NLT}_{\mathrm{DN}}$ indicates the DN values of the NLT products; $K_{2000}$ is the ratio between population and $\mathrm{DN}$ values in 2000; $r$ indicates the population increase rate from 2000 to 2010.

2.3.4 Precision Validation: The precision validation was executed by comparing the estimated population and census population of each city. A relative errors (RE) indicator was applied (Function(6)).

$$
\mathrm{RE}=\frac{\mid \text { Estimated population-Census population } \mid}{\text { Census population }} \times 100 \%
$$

2.3.5 Analysis of Population Evolution: The population spatialization in 2005 provided opportunities to monitor population evolution on the long sequence and multi frequency. The population dynamics was monitored and compared at city scales in this work. Various total numbers of population could reflect spatial characteristics of population distribution in the PRD, while different increase numbers were able to show population dynamics from 2000 to 2010 .

\subsection{Result}

2.4.1 Evaluation of the Estimated Population: By comparing the estimated population and census population of each city, the precision of population spatialization method was validated (Figure 2). Obviously, the bar graph showed a consistent dynamics of the estimated population and the census population in nine cities of the PRD (Figure 2(a)). The bar heights of estimated population and census population even exhibited little differences.

In addition, the relative errors in 2000 and 2010 were less than $0.01 \%$, implying the high accuracy of population spatialization method in census years (Figure 2(b)). However, the relative error in 2005 ranged from $5.90 \%$ to $24.11 \%$, which were distinctly larger than that in 2000 and 2010 (Figure (b)). In this work, the estimated population in non-census years was calculated on basis of the estimated results in census years. Hence, the estimated result in 2005 was mainly influenced by the census data, especially the fifth census data bulletin. As illustrated in many literatures, the fifth national census data may be larger than the real population due to its special statistic approach about leakage rate, resulting in a relatively high computational base. For instance, the census population in 2000 was even larger than the $1 \%$ population sample surveys in 2005. Therefore, the overestimated result in 2005 was relatively reasonable.

Totally, the population spatialization method is feasible to obtain precise population distribution information at pixel scales.
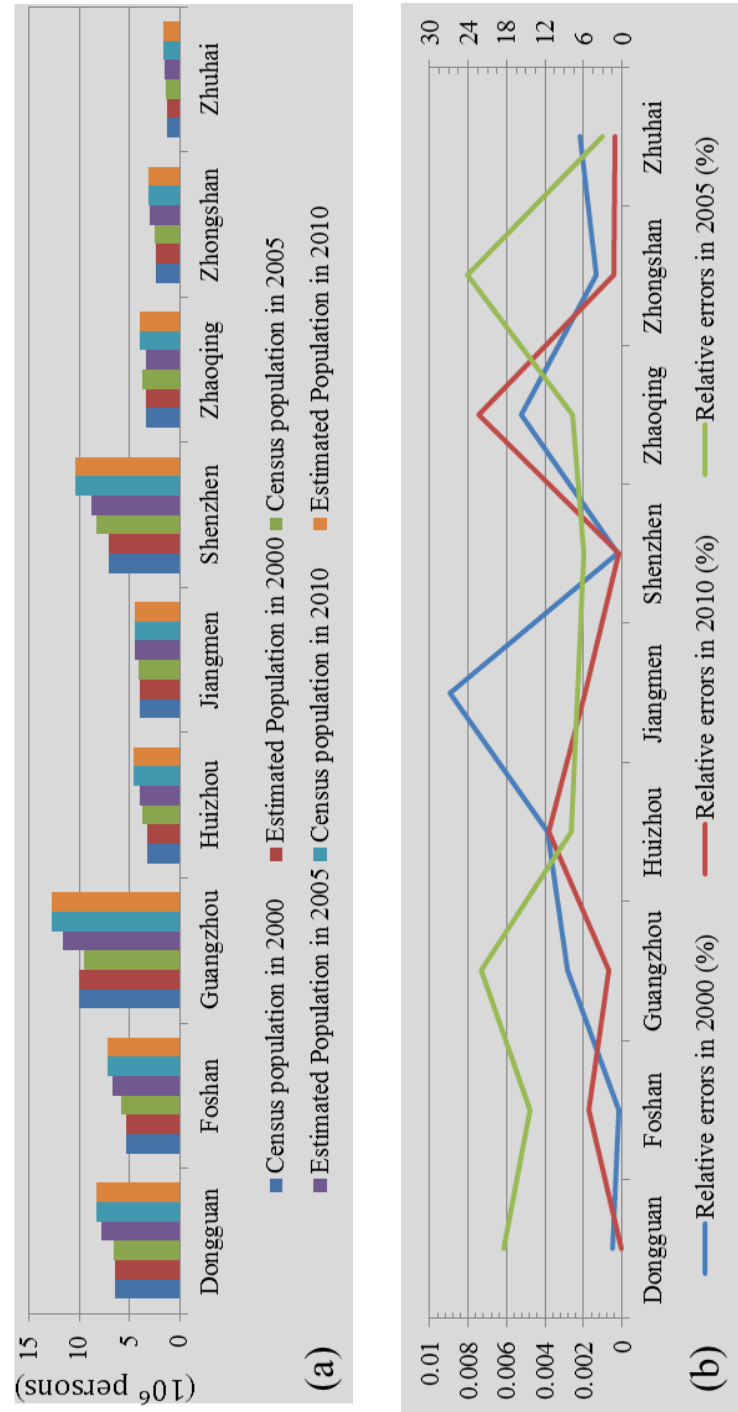

Figure 2. The comparison between census population and estimated population from 2000 to 2010, including (a) population in each city and (b) relative errors in each city

2.4.2 Population Evolution in the PRD from 2000 to 2010: According to the estimation in this work, in 2000, total population in Guangzhou was the largest, followed by Shenzhen, Dongguan, Foshan, Jiangmen, Zhaoqing, Huizhou, Zhongshan and Zhuhai (Figure 3). Total population in Guangzhou was about 8.05 times of that in Zhuhai. However, population density of the nine cities did not exhibit the same order in consideration of the various jurisdiction areas. Furthermore, Shenzhen and Dongguan were the first two densest cities with the population density of 3213 and 3020 persons per $\mathrm{km}^{2}$, respectively. Zhongshan, Guangzhou, Foshan and Zhuhai were another four denser cities with the population density of 1452, 1443, 1343 and 1047 persons per $\mathrm{km}^{2}$, respectively. Although Jiangmen, Huizhou and Zhaoqing possessed the largest areas among the nine cities in the PRD, they showed the sparsest average population densities of 412, 281 and 232 persons per $\mathrm{km}^{2}$ in 2000, which were far lower than the regional average. Population density in Shenzhen was nearly 13.82 times of that in Zhaoqing. 

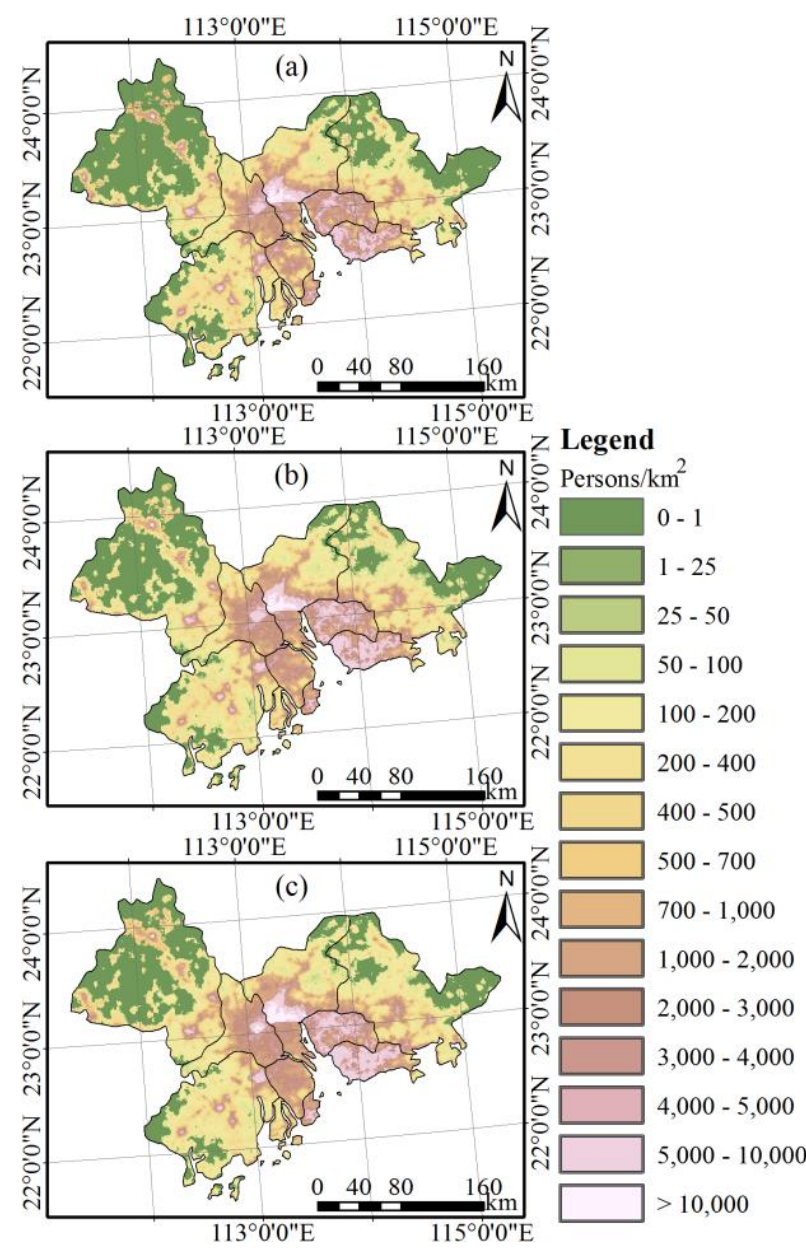

Figure 3. Estimated population at $1 \mathrm{~km} * 1 \mathrm{~km}$ scales from 2000 to 2010

From 2000 to 2005, total population in nine cities of the PRD showed increase trend (Figure 3). The increased population ranged from 17033 persons (Zhaoqing) to 1757420 persons (Shenzhen). Total population in Huizhou surpassed that in Zhaoqing. Different population increase resulted in various dynamics of population density. However, it did not influence the population density order. Totally, Shenzhen was the densest city with population density larger than 4000 persons per km2, Dongguan was the second populous city with 3644 persons per km2, followed by Zhongshan, Guangzhou, Foshan, Zhuhai, Jiangmen, Huizhou and Zhaoqing with population density between 234 persons per $\mathrm{km} 2$ to 1856 persons per $\mathrm{km} 2$. Until 2005, population density in Shenzhen was nearly 15.60 times of that in Zhaoqing.

From 2005 to 2010, total population in nine cities of the PRD kept increasing, but the increase speed turned slower than that from 2000 to 2005 (Figure 3). Shenzhen showed the fastest increase with 318432 persons per year, followed by Guangzhou, Huizhou, Foshan, Zhaoqing, Dongguan, Zhuhai, Zhongshan and Jiangmen with increase speed ranging from 13127 persons per year to 224247 persons per year. Total population in Huizhou surpassed that in Jiangmen. Population density of the nine cities continuously increased. However, the population density order of the nine cities did not change. That is to say, Shenzhen remained the most populous city and Zhaoqing were still the sparest. In 2010, Shenzhen showed the highest population density of 4749 persons per $\mathrm{km}^{2}$, which was about 17.60 times of that in Zhaoqing.
Therefore, the largest total population was located in Guangzhou, whereas the smallest total population was in Zhaoqing from 2000 to 2010. The total population of Huizhou showed faster increase than Zhaoqing in 2000-2005 and Jiangmen in 2005-2010. In comparison, population increase in the PRD during 2000-2005 was faster than that in 2005-2010. At the same time, population density in the PRD showed increase trend from 2000 to 2010, but it was denser in the central region than that in the northern, eastern and western regions.

\section{CONCLUSIONS}

Based on the NLT products, MOD09A1 products, census population and $1 \%$ population sample surveys data, population in the PRD from 2000 to 2010 were spatialized into per $\mathrm{km}^{2}$. Nine cities in the PRD were selected as the study area, and the detailed population dynamics were monitored and analyzed. Results showed several conclusions:

(1) The proposed REEI applied the NDVI to impair light saturation and blooming in the PRD, applied the MNDWI to avoid overcorrection of the NDVI and applied the NMDBI to enhance DN values of urban pixels, resulting in relatively similar light with the actual resident extent. The REEI presented more superiority in impairing light blooming and separating residential area from the NLT products.

(2) The population spatialization, especially in non-census years like 2005, provided a good opportunity to monitor population evolution at intervals of five years. The relative errors in 2000 and 2010 were less than $0.01 \%$. The total relative error in 2005 was about $12.18 \%$, which is influenced by the census data, especially the fifth census data bulletin. Results showed that the population spatialization method in census year was feasible and that in the non-census year was reasonable. Hence, the methods proposed in this work to obtain precise population distribution information at pixel scales were feasible.

(3) During the period of 2000-2010, Guangzhou, Shenzhen, Dongguan and Foshan were top four cities with larger total population due to their convenient transportation and abundant fishery resources. Zhongshan and Zhuhai loaded less population than other cities. Due to various city areas, population density didn't exhibit the same order. Population was denser in the central PRD, such as Shenzhen, Guangzhou, Zhongshan, Foshan, Dongguan and Zhuhai. However, other cities, including Zhaoqing in the northern PRD, Huizhou in the Eastern PRD and Jiangmen in the western PRD, occupied larger areas but loaded sparser population during the period of 2000-2010.

(4) During the study decade, population increase and expansion presented various characteristics in different cities of the PRD. In comparison, population in the PRD increased faster in 2000-2005 than 2005-2010. Population in Huizhou exceeded that in Zhaoqing in 2005 and Jiangmen in 2010, respectively. Shenzhen showed fastest population increase whereas Zhuhai showed slowest population increase. Totally, from 2000 to 2010, population distribution presented wider coverage and denser population in most pixels, especially along the Pearl River.

\section{ACKNOWLEDGEMENTS}


This research was supported by the International Partnership Program of Chinese Academy of Sciences [grant number 131C11KYSB20160061].

\section{REFERENCES}

Elvidge, C. D., Baugh, K. E., Dietz, J. B., Bland, T., Sutton, P. C., \& Kroehl, H. W., 1999. Radiance calibration of DMSP-OLS low-light imaging data of human settlements. Remote Sensing of Environment, 68(1), pp. 77-88.

Lo, C. P., 2001. Modeling the population of china using dmsp operational linescan system nighttime data. Photogrammetric Engineering \& Remote Sensing, 67(9), pp. 1037-1048.

Malthus, T. R., 2008. Population theory. Peking University Press, Beijing.

Sun, W., Zhang, X., Wang, N., Cen, Y., 2017. Estimating population density using DMSP-OLS night-time imagery and land cover data. IEEE Journal of Selected Topics in Applied Earth Observations \& Remote Sensing, 10(6), pp. 2674-2684.

Sutton, P. 1997. Modeling population density with night-time satellite imagery and GIS. Computers Environment \& Urban Systems, 21(3-4), pp. 227-244.

Tripathy, B. R., Tiwari, V., Pandey, V., Elvidge, C. D., Rawat, J. S., Sharma, M. P., Prawasi, R., Kumar, P., 2017. Estimation of urban population dynamics using dmsp-ols night-time lights time series sensors data. IEEE Sensors Journal, 17(4), pp. 1013-1020.

Wei, C., Taubenböck, H., Blaschke, T., 2017. Measuring urban agglomeration using a city-scale dasymetric population map: a study in the pearl river delta, china. Habitat International, 59, pp. 32-43.

Yang, Q.; Li, L.; Wang, Y.; Wang, X.; Lu, Y., 2016. Spatial distribution pattern of population and characteristics of its evolution in China during 1935-2010. Geogr. Res., 35(8), $1547-1560$.

You, Z., Wang, L., Feng, Z. M., Yang, Y. Z., 2013. Spatio-temporal pattern and variantion characteristics of population distribution at county level in the Pearl River Delta. Tropical Geography, 33(2), pp. 156-163.

Yu, S., Zhang, Z. and Liu, F., 2018. Monitoring Population Evolution in China Using Time-Series DMSP/OLS Nightlight Imagery. Remote Sensing, 10, pp. 194.

Zeng, C.; Zhou, Y.; Wang, S.; Yan, F.; Zhao, Q., 2011. Population spatialization in China based on night-time imagery and land use data. Int. J. Remote Sens. 32(24), pp. 9599-9620.

Zhang, Q., Schaaf, C., Seto, K. C., 2013. The vegetation adjusted NTL urban index: a new approach to reduce saturation and increase variation in nighttime luminosity. Remote Sensing of Environment, 129(2), pp. 32-41.

Zhao, N., Zhou, Y., Samson, E. L., 2014. Correcting incompatible DN values and geometric errors in nighttime lights time-series images. IEEE Transactions on Geoscience \& Remote Sensing, 53(4), pp. 2039-2049.
Zhuo, L., Zheng, J., Zheng, J., Chen, J., Shi, P. J., Li, X. 2009. Modelling the population density of china at the pixel level based on DMSP/OLS non-radiance-calibrated night-time light images. International Journal of Remote Sensing, 30(4), $1003-1018$ 